extracellular signal-regulated kinases (ERKs), c-Jun-N-terminal kinases (JNKs) and p38) are key players in RA. Using human tumour necrosis factor transgenic (hTNFtg) mice as a model for inflammatory arthritis, we have already characterised the role of JNK1 and p38 $\alpha$ in regulating TNF $\alpha$-induced arthritis. We now investigate the role of the ERK-activated kinase ribosomal S6 protein kinase 2 (RSK2).

Material and methods hTNFtg mice lacking RSK2 (hTNFtg;rsk2-/y) were generated. The effects of RSK2 inactivation on the development of clinical signs of arthritis (body weight loss, paw swelling and grip strength) were analysed. Local inflammation, cartilage and bone loss as well as osteoclast numbers were quantifed by histomorphometry of the paws. Systemic bone loss was analysed by micro-CT of tibia, dynamic bone histomorphometry, quantification markers for bone turnover, quantitative PCRs for bone formation and resorption markers. In vitro osteoclast differentiation was performed to compare the response of osteoclast progenitors to TNF $\alpha$.

Results Earlier onset of arthritis and worsening of clinical signs of arthritis indicated a sensitisation to TNF-induced arthritis in the hTNFtg;rsk2-/y mice. We confirmed the diagnosis histologically by showing increased synovitis, cartilage and bone destruction as well as increased osteoclast numbers in the inflamed joints. Cartilage destruction was associated with lower collagen 2 expression, a chondrocyte marker, and higher levels of matrix metalloproteinase 13, a cartilage degrading enzyme. A drastically reduced bone mass in the absence of RSK 2 was found by micro-CT and by histomorphometry, demonstrating a quasi-absence of trabecular bone and a striking decrease in cortical bone. Increased osteoclast numbers per bone surface and an almost absence of osteoblasts (the bone forming cells) characterised the phenotype. However, no intrinsic osteoclast differentiation defect in response to macrophage colony-stimulating factor and RANKL or to TNF $\alpha$ was observed. Decreased levels of circulating osteocalcin, a marker for bone formation, and dynamic histomorphometry confirmed the almost absence of bone formation. Quantitative PCRs revealed decreased expression levels of genes essential for osteoblast differentiation such as osterix or characterising osteoblast activity like collagen 1 and osteocalcin in the long bones of hTNFtg;rsk2-/y mice.

Conclusion In addition to exerting a protective effect against local joint destruction, the ERK-activated RSK2 kinase appears to oppose the local bone destructive effect of TNF $\alpha$ by protecting bone formation by osteoblasts. These observations open new perspectives for the development of small molecules targeting ERK-RSK2 for regenerative treatment of inflammatory arthritis.

\title{
A60 ERK-ACTIVATED KINASE RSK2 PROTECTS AGAINST INFLAMMATORY ARTHRITIS-INDUCED BONE DESTRUCTION BY OPPOSING THE TUMOUR NECROSIS FACTOR $\alpha$-MEDIATED INHIBITION OF BONE FORMATION
}

C Böhm, M M Zaiss, R Axmann, A Hess, K Engelke, G Kollias, G Schett, J-P David Department of Medicine 3, Rheumatology and Immunology, University of Erlangen-Nuremberg, Germany

\subsection{6/ard.2010.129593v}

Background Local destruction of cartilage and bone of joints followed by systemic osteopenia characterise rheumatoid arthritis (RA). Proinflammatory cytokines like interleukin $\beta$ (IL $\beta$ ) and tumour necrosis factor $\alpha$ (TNF $\alpha$ ) known to activate all major mitogen-activated protein kinases (MAPKs; 